Article

\title{
Acylation of Heteroaromatic Amines: Facile and Efficient Synthesis of a New Class of 1,2,3-Triazolo[4,5-b]pyridine and Pyrazolo[4,3-b]pyridine Derivatives
}

\author{
Hamada Mohamed Ibrahim ${ }^{1,2}$, Haider Behbehani ${ }^{1, *}$, Saad Makhseed ${ }^{1}$ and \\ Mohamed H. Elnagdi ${ }^{1}$
}

1 Chemistry Department, Faculty of Science, Kuwait University, P.O. Box 5969, Safat 13060, Kuwait

2 Chemistry Department, Faculty of Science, Fayoum University, Fayoum, A. R., Egypt

* Author to whom correspondence should be addressed; E-Mail: hbehbehani@live.com; Tel.: +965-990-630-32; Fax: +965-248-164-82.

Received: 14 March 2011; in revised form: 21 April 2011 / Accepted: 26 April 2011 /

Published: 4 May 2011

\begin{abstract}
Triazolo[4,5-b]pyridines and pyrazolo[4,3-b]pyridines can be readily prepared via cyanoacetylation reactions of 5-amino-1,2,3-triazoles 1a,b and 4-aminopyrazole 2 followed by subsequent cyclization of the formed cyanoacetamides. Reactions of amines 1a,b with a mixture of $p$-nitrophenylacetic acid and acetic anhydride under microwave irradiation conditions afforded the corresponding amides 15a,b that underwent cyclization to form 1,2,3-triazolo[4,5-b]pyridines 16a,b upon heating in DMF solutions containing sodium acetate. Reactions of 1a,b with active methylene compounds, including 17a-c, in the presence of zeolites as catalyst also afforded 1,2,3-triazolo[4,5-b]pyridine derivatives 20a-f via the intermediacy of triazole derivatives 19 and not 18.
\end{abstract}

Keywords: cyanoacetic acid; cyanoacetamides; triazolo[4,5-b]pyridine; pyrazolo[4,3-b]pyridine; $p$-nitrophenylacetic acid; zeolite

\section{Introduction}

Pyrazolo[4,3-b]pyridine and triazolo[4,5-b]pyridine derivatives are of interest for their various applications as vasodilators, hypotensive, hypoglycemic, anti-inflammatory, analgesic, antiasthmatic, 
antipyretic agents and as substrates of NAD glycohydrolase [1-3]. Owing to these interesting biological activities and medicinal properties these azolopyridine derivatives have been the targets of investigations by several research groups [4-8]. The present study describes the results of an investigation aimed at the preparation of a new class of 1,2,3-triazolo[4,5-b]pyridine and pyrazolo[4,3$b$ ]pyridine derivatives. Cyanoacetylation of electron rich aromatic compounds and heteroarmatic amines, initially described by Slatt et al., [9] has found extensive utility in efficient routes for the preparation of 3-oxoalkanonitriles [10-15] and cyanoacetamides [16-18]. Cyanoacetylation of $o$-acyl heteroarmatic amines is expected to give cyanoacetamides in which an active methylene moiety is located in close proximity to a ketone carbonyl function. This enables ready cyclization of the products to form fused pyridines. However, to our knowledge this synthetic approach has not been explored to date. Below, we describe the results of an investigation of the preparation of cyanoacetamides and arylacetamides of 5-amino-1,2,3-triazoles and 4-aminopyrazoles and their utility in the preparation of condensed pyridines.

\section{Results and Discussion}

Readily obtainable (5-amino-2-phenyl-2H-1,2,3-triazol-4-yl)phenylmethanone (1a) [19], 1-(5amino-2-phenyl-2H-1,2,3-triazol-4-yl)ethanone (1b) [20] and 4-amino-3-benzoyl-1-phenyl- $1 H$ pyrazole-5-carbonitrile (2) [21] were found to react with a preheated mixture of acetic anhydride and cyanoacetic acid under microwave irradiation conditions to yield the corresponding cyanoacetamides 3 and 4 in excellent yields. These substances undergo cyclization to generate the respective fused pyridones $\mathbf{5}$ and $\mathbf{6}$ upon stirring at reflux for $30 \mathrm{~min}$ in DMF containing anhydrous sodium acetate (cf. Scheme 1). The structure of 5a was assigned by using X-ray crystallographic analysis (cf. Table 1 and Figure 1).

Scheme 1. Reaction of 5-amino-1,2,3-triazoles and 4-aminopyrazole with cyanoacetic acid.

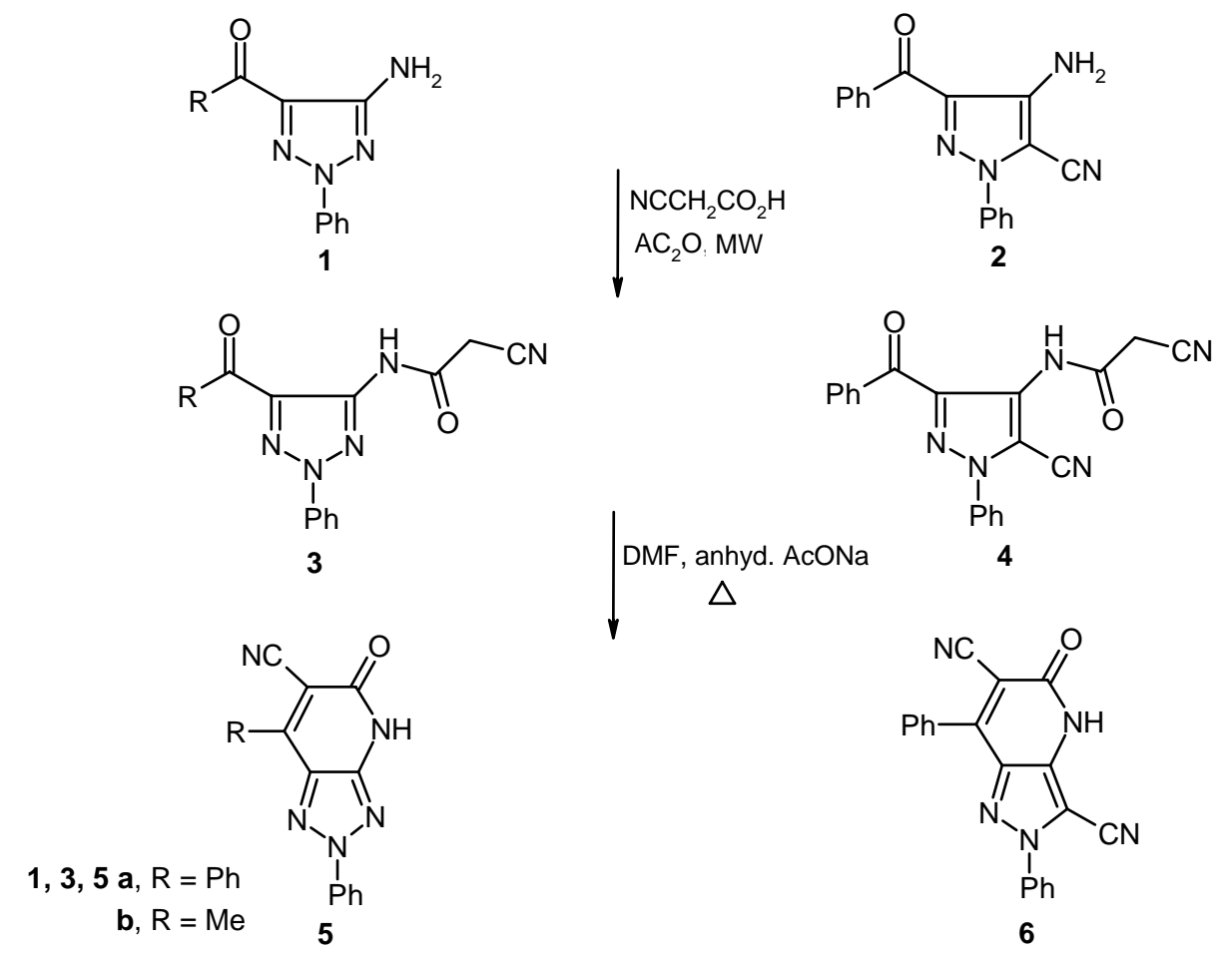


Figure 1. ORTEP plot of the x-ray crystallographic data determined for 5a. Crystallographic data have been deposited with the Cambridge Crystallographic Data Centre as supplementary publication number CCDC 804721 [22].

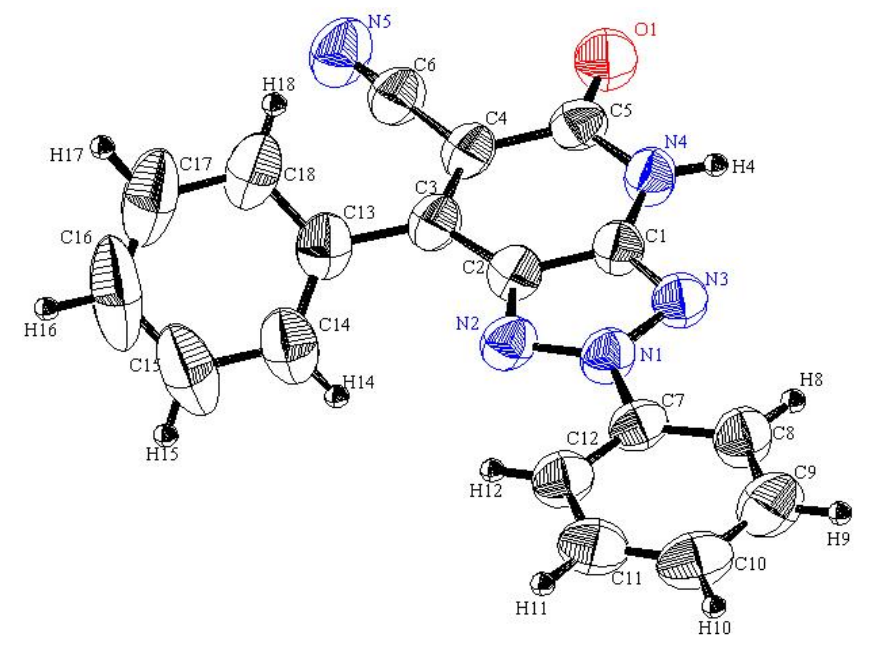

Table 1. Selected bond lengths and bond angles for $5 \mathbf{a}$.

\begin{tabular}{lccc}
\hline Bond & Bond length & Bond & Bond angle \\
\hline N1-N2 & 1.327 & N1-N2-C2 & 102.5 \\
N1-N3 & 1.356 & N1-N3-C1 & 100.4 \\
N3-C1 & 1.333 & N2-N1-N3 & 117.5 \\
N2-C2 & 1.350 & N3-C1-C2 & 111.6 \\
C1-C2 & 1.384 & N2-C2-C1 & 108.0 \\
C1-N4 & 1.375 & C1-C2-C3 & 121.4 \\
\hline
\end{tabular}

The acylpyrazole 7 underwent ready cyanoacylation to afford the cyanoacetamide 8 in $93 \%$ yield. Heating a solution of $\mathbf{8}$ in DMF containing anhydrous sodium acetate leads to production of a substance whose structure should be either $\mathbf{9}$ or its isomer $\mathbf{1 0}$.

The actual structure of the product was assigned as $\mathbf{1 0}$ based on its ${ }^{13} \mathrm{C}-\mathrm{NMR}$ spectroscopic data which showed the absence of an acetyl carbonyl carbon resonance and its replacement by a peak at $186.19 \mathrm{ppm}$. Moreover, the methyl protons' resonance at $\delta=2.12 \mathrm{ppm}$ displays a HMBC cross peak with the carbon peak at $\delta=105.72 \mathrm{ppm}$ that is assigned as C- 6 . In addition the X-ray crystallographic analysis of this product demonstrated that it has the structure represented by $\mathbf{1 0}$ (cf. Scheme 2 and Figure 2).

In a similar manner, bis-acetylpyrazole $\mathbf{1 1}$ is readily cyanoacylated to afford the corresponding cyanoacetamide 12 in excellent yield. Heating a DMF solution of 12 containing anhydrous sodium acetate afforded a product that may also have the isomeric structures represented by $\mathbf{1 3}$ and $\mathbf{1 4}$. As before, the actual structure of the product was shown to be $\mathbf{1 4}$ based on its ${ }^{13} \mathrm{C}$-NMR spectrum, which contained a carbonyl resonance at $202.41 \mathrm{ppm}$. This chemical shift is expected for an acyl carbonyl at the $\mathrm{C}-3$ position of the pyrazole ring and not at C-5 since in the latter case shielding provided by the $\mathrm{N}$-lone pair should make the resonance appear at a higher field (cf. Scheme 3). 
Scheme 2. Synthesis of pyrazolo[4,3- $b]$ pyridine-6-carbonitrile $\mathbf{1 0}$.<smiles>CC(=O)c1c(N)c(C(=O)O)nn1-c1ccccc1</smiles>

7<smiles>CC(=O)c1c(NC(=O)CC#N)c(C(=O)c2ccccc2)nn1-c1ccccc1</smiles>

8<smiles>CC(=O)c1c2[nH]c(=O)c(C#N)c(-c3ccccc3)c2nn1-c1ccccc1</smiles>

Figure 2. ORTEP plot of the x-ray crystallographic data determined for $\mathbf{1 0}$ containing one DMSO molecule. Crystallographic data have been deposited in the Cambridge Crystallographic Data Centre as supplementary publication number CCDC 816562 [23].

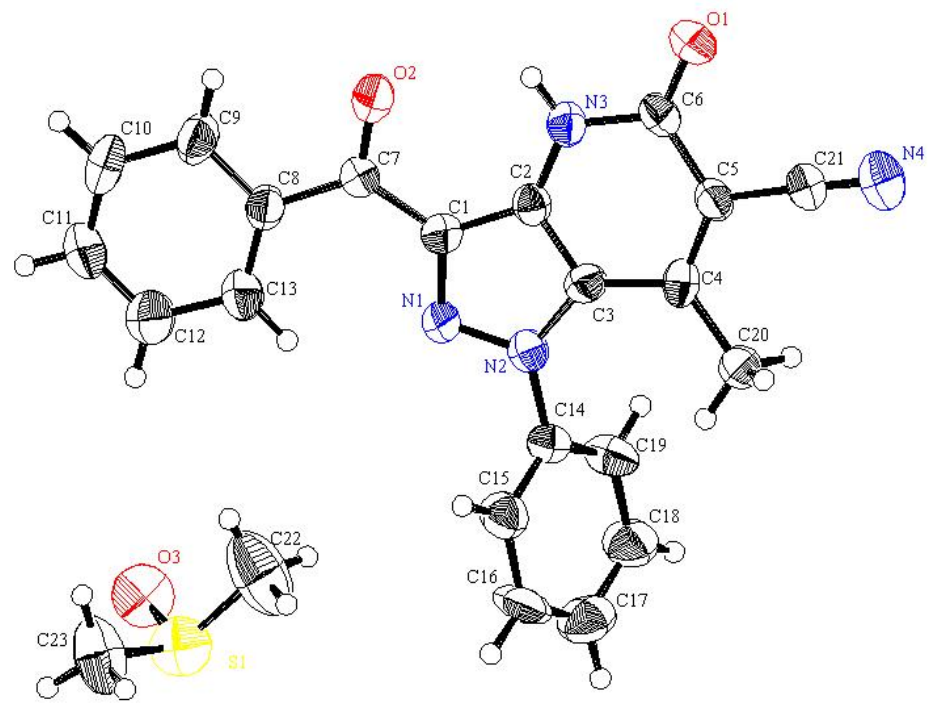

Scheme 3. Synthesis of pyrazolo[4,3-b]pyridine-6-carbonitrile 14.<smiles>CCC(C(C)=O)C(=O)Nc1c(C(C)=O)nn(-c2ccccc2)c1C(C)=O</smiles> 
We have previously [10] suggested that in the mechanistic pathway for the process described by Slatt [9], the mixed anhydride is formed initially and then it reacts by nucleophilic addition of an amine or electron rich aromatic system at the more electron deficient cyanoacetyl carbonyl. As a consequence of this proposal, we believed that other mixed anhydrides could be used as arylacetamide precursors provided that the reactions occur at the more electron deficient aroyl carbonyl. In fact, heating $p$-nitrophenylacetic acid with acetic anhydride, followed by addition of either $\mathbf{1 a}$ or $\mathbf{1 b}$ and heating the mixture in a microwave oven for $60 \mathrm{~s}$, afforded the corresponding amides 15a and 15b that are readily cyclized to form the respective triazolo[4,5-b]pyridines derivatives $\mathbf{1 6 a}$ and $\mathbf{1 6} \mathbf{b}$ upon heating in DMF containing anhydrous sodium acetate (cf. Scheme 4).

Scheme 4. Reaction of 5-amino-1,2,3-triazoles with $p$-nitrophenylacetic acid.

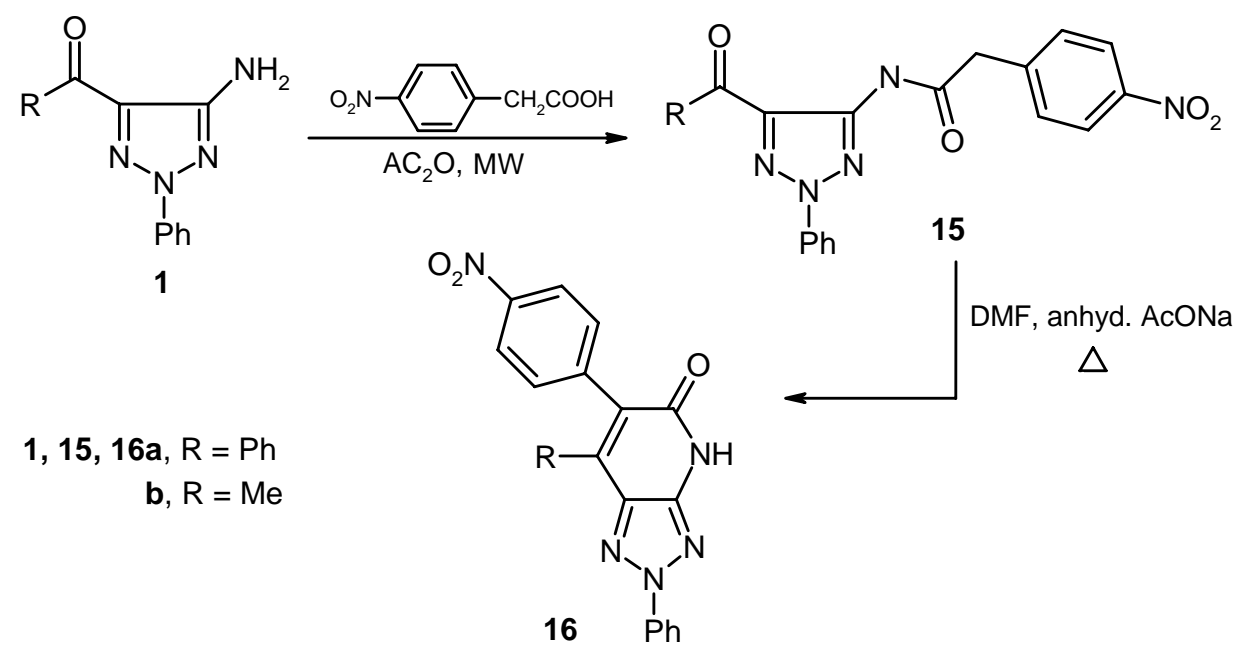

We have explored a possible extension of this methodology, which relies on conversion of the acetamide derivatives of 1,2,3-triazoles to their corresponding 1,2,3-triazolo[4,5- $b$ ]pyridines, by probing the reactivity 1a,b with other active methylene compounds like 17a-c. The results of this study showed that 1a,b underwent condensation with 17a-c in presence of zeolite catalysts, followed by heating to produce the corresponding 1,2,3-triazolo[4,5-b]pyridine derivatives 20a-f. The structure of $20 \mathrm{f}$ was assigned by X-ray crystallographic analysis. Although these products could potentially formed via the intermediacy of either triazole $\mathbf{1 8}$ or $\mathbf{1 9}$, it is almost certain that $\mathbf{1 9}$ is the intermediate as attempts to condense $N$-acetyl-1,2,3-triazole derivatives 21 with active methylene compounds failed. Moreover the reaction of 20a with another molecule of 1a afforded 22, which is generated via elimination of ethanol (cf. Figure 3 and Scheme 5).

In contrast, $\mathbf{1 b}$ was found to react with ethyl acetoacetate (17b) in absence of zeolite to yield a condensation product that arises by elimination of one molecule of water. X-ray crystallographic analysis of this substance demonstrated that it has the structure represented by 23, a product that is formed via initial addition of the amine to the carbonyl carbon of $\mathbf{1 7 b}$ (Figure 4, Table 2). The fact that 1b reacts with ethyl acetoacetate $(\mathbf{1 7 b})$ to yield either the intermediate 23 or $\mathbf{1 9 f}$ demonstrates the effect of the zeolite, a microporous catalyst that favors formation of slim molecules like $\mathbf{1 9}$ rather than bulky ones like 23, so the latter is formed in absence of such a catalyst, Also, 23 separated from the reaction mixture underwent cyclization in refluxing DMF containing anhydrous sodium acetate to form 24 via loss of another molecule of water. (cf. Scheme 6). 
Figure 3. ORTEP plot of the x-ray crystallographic data determined for 20f. Crystallographic data have been deposited in the Cambridge Crystallographic Data Centre as supplementary publication number CCDC 815380 [24].

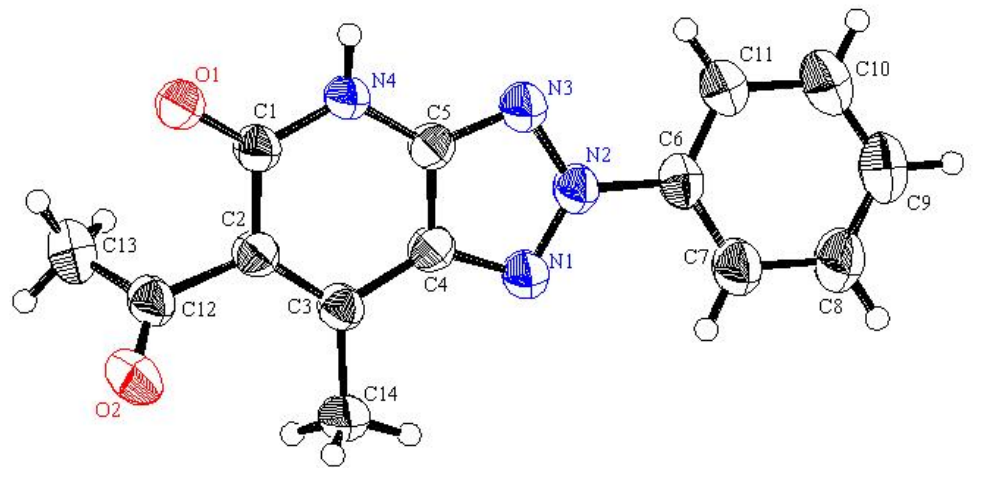

Scheme 5. Reaction of 5-amino-1,2,3-triazoles with active methylene compounds.<smiles>[Y]C(=O)Nc1nn(-c2ccccc2)nc1C([R])=O</smiles>

\begin{tabular}{r|ll}
$\mathbf{2 0}$ & $\mathbf{X}$ & $\mathbf{R}$ \\
\hline $\mathbf{a}$ & $\mathrm{COOEt}$ & $\mathrm{Ph}$ \\
$\mathbf{b}$ & $\mathrm{PhCO}$ & $\mathrm{Ph}$ \\
$\mathbf{c}$ & $\mathrm{CH}_{3} \mathrm{CO}$ & $\mathrm{Ph}$ \\
$\mathbf{d}$ & $\mathrm{COOEt}$ & $\mathrm{CH}_{3}$ \\
$\mathbf{e}$ & $\mathrm{PhCO}$ & $\mathrm{CH}_{3}$ \\
$\mathbf{f}$ & $\mathrm{CH}_{3} \mathrm{CO}$ & $\mathrm{CH}_{3}$
\end{tabular}<smiles>C[14C](C)[14CH]1C(=O)Nc2nn(-c3ccccc3)nc21</smiles>

22<smiles>[Y]c1c([Y])c2nn(-c3ccccc3)nc2[nH]c1=O</smiles>

20

Scheme 6. Reaction of $\mathbf{1 b}$ with ethyl acetoacetate to afford 24.

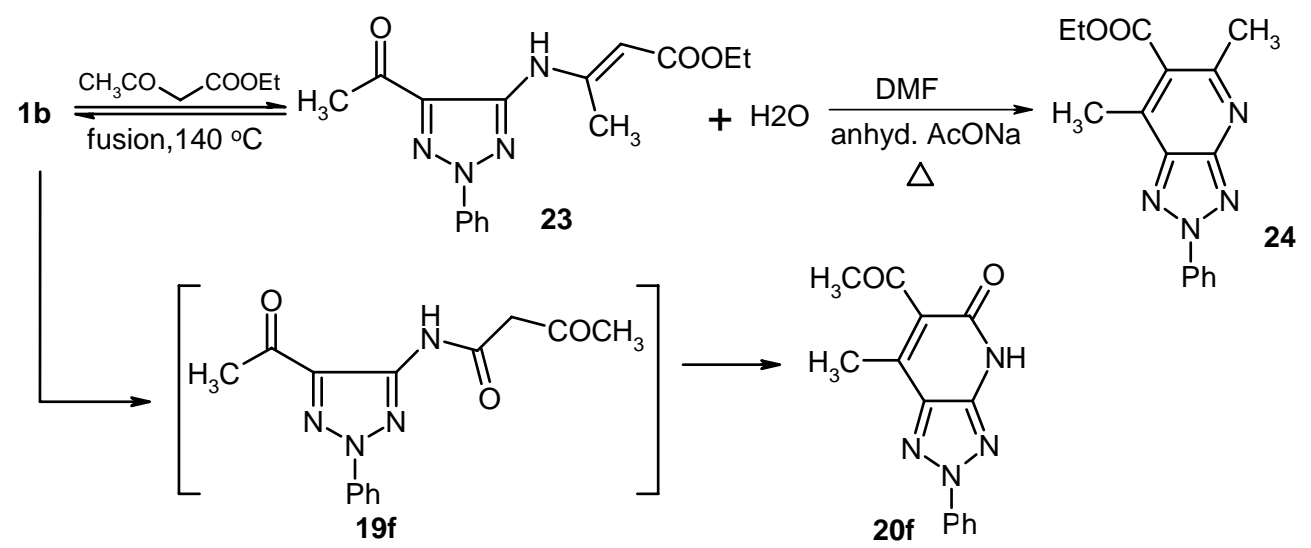


Figure 4. ORTEP plot of the x-ray crystallographic data determined for 23. Crystallographic data have been deposited in the Cambridge Crystallographic Data Centre as supplementary publication number CCDC 805282 [25].

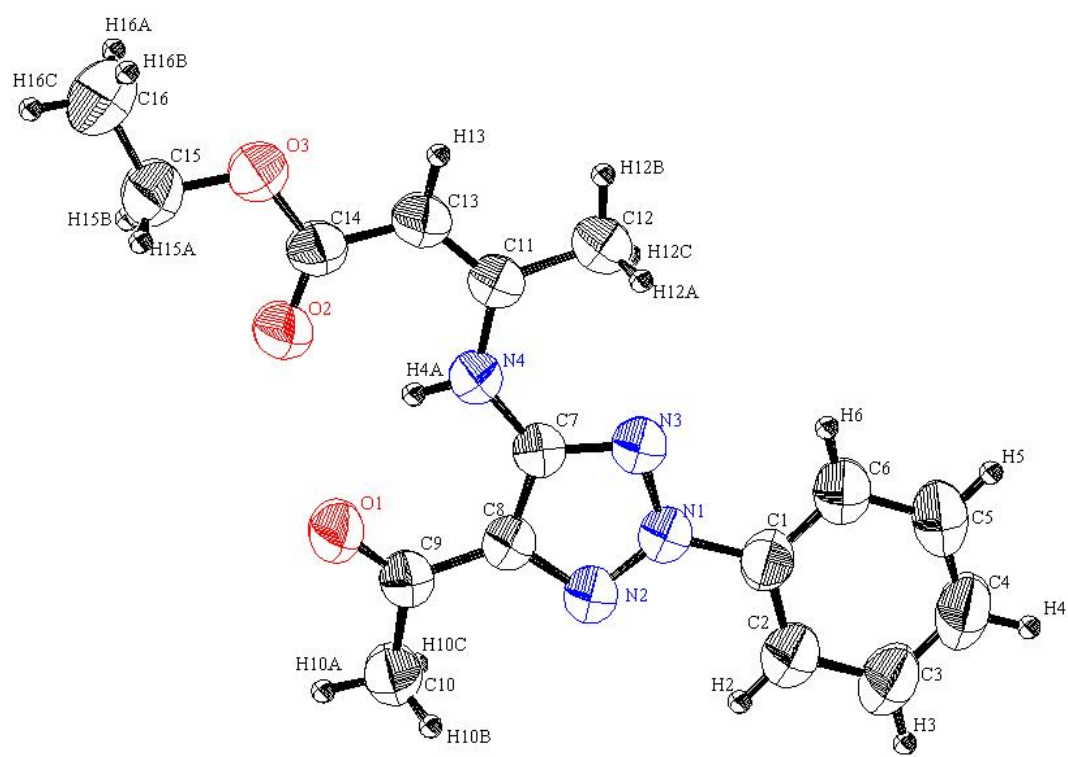

Table 2. Selected bond lengths and bond angles for 23.

\begin{tabular}{cccc}
\hline Bond & Bond length & Bond & Bond angle \\
\hline N1-N2 & 1.317 & N1-N2-C8 & 103.8 \\
N1-N3 & 1.359 & N1-N3-C7 & 102.7 \\
N3-C7 & 1.330 & N2-N1-N3 & 116.1 \\
N2-C8 & 1.336 & N3-C7-C8 & 109.0 \\
C8-C9 & 1.462 & N2-C8-C7 & 108.5 \\
N4-C7 & 1.379 & O1-C9-C8 & 119.5 \\
\hline
\end{tabular}

Inspection of the crystallographically determined bond angles and lengths of the 1,2,3-triazole rings in both 5a, 20f and 23 indicate that the N3-N1-N2 bond angles deviate significantly from typical sp ${ }^{3}$ nitrogen values and are close to those that are associated with $\mathrm{sp}^{2}$ nitrogens. However, the N2-C8-C7 or N3-C7-C8 bond angles are close to those expected for $\mathrm{sp}^{3}$ carbons. Similar observations, made earlier in studies of very similar systems by Elnagdi et al., [19] have been taken as evidence for the significant contribution of charge separated resonance forms delocalizing N-1 lone pairs to the ring carbons. Importantly, both 5a, 20 and 23 are planar substances, a fact that adds further support to the conclusion that resonance delocalization of $\mathrm{N}-1$ lone pair occurs in these systems.

\section{Experimental}

\subsection{General}

Melting points were recorded on a Griffin melting point apparatus and are reported uncorrected. IR spectra were recorded using KBr disks using a Perkin-Elmer System 2000 FT-IR spectrophoto- meter. ${ }^{1} \mathrm{H}-\mathrm{NMR}(400 \mathrm{MHz})$ and ${ }^{13} \mathrm{C}-\mathrm{NMR}(100 \mathrm{MHz})$ spectra were recorded at $25{ }^{\circ} \mathrm{C}$ in $\mathrm{CDCl}_{3}$ or DMSO- $d_{6}$ as solvent with TMS as internal standard on a Bruker DPX 400 super-conducting NMR spectrometer. 
Chemical shifts are reported in ppm. Mass spectra were measured using a high resolution GC-MS (DFS) thermo spectrometers with EI (70 EV). Microanalyses were performed on a LECO CHNS-932 Elemental Analyzer. Reactions were conducted under microwave irradiation in heavy-walled Pyrex tubes (capacity $10 \mathrm{~mL}$ ) fitted with PCS caps. Microwave heating was carried out with a single mode cavity Explorer Microwave synthesizer (CEM Corporation, 3100 Smith Farm Road, Matthews, NC, USA). The zeolite $(\leq 45 \mu \mathrm{m})$ was purchased from Fluka Company with product No. 96096. The crystal structures were determined by a Rigaku R-AXIS RAPID diffractometer using filtered Mo-K $\alpha$ radiation at Kuwait University. Compounds 1a,b, 2 and 21 were prepared using literature procedures [19-21].

\subsection{General Procedure for the Preparation of Cyanoacetamides 3, 4, 8 and 12}

A solution of cyanoacetic acid $(0.45 \mathrm{~g}, 5 \mathrm{mmol})$ in $\mathrm{Ac}_{2} \mathrm{O}(5 \mathrm{~mL})$ was heated in the microwave oven at $85^{\circ} \mathrm{C}$ for $10 \mathrm{~s}$ then compounds $1,2,7$ or $11(5 \mathrm{mmol})$ were added and the reaction mixture was heated for further $30 \mathrm{~s}$ at $100{ }^{\circ} \mathrm{C}$. The reaction mixture was allowed to cool to room temperature and the formed crystalline solid was separated by filtration and washed with cold ethanol and then hot ethanol to afford $3, \mathbf{4 , 8}$ and $\mathbf{1 2}$, respectively, as pure substances.

N-(5-Benzoyl-2-phenyl-2H-1,2,3-triazol-4-yl)-2-cyanoacetamide (3a). Creamy white crystals, yield: 98\%, m.p. $210{ }^{\circ} \mathrm{C}$; IR (KBr): v/cm ${ }^{-1} 3291(\mathrm{NH}), 2257$ (CN), 1692, 1634 (2CO); ${ }^{1} \mathrm{H}-\mathrm{NMR}$ (DMSO$\left.d_{6}\right): \delta=4.04\left(\mathrm{~s}, 2 \mathrm{H}, \mathrm{CH}_{2}\right), 7.50(\mathrm{t}, J=7.6 \mathrm{~Hz}, 1 \mathrm{H}, \mathrm{Ar}-\mathrm{H}), 7.56-7.64(\mathrm{~m}, 4 \mathrm{H}, \mathrm{Ar}-\mathrm{H}), 7.73(\mathrm{t}, J=7.6 \mathrm{~Hz}$, $1 \mathrm{H}, \mathrm{Ar}-\mathrm{H}), 8.01(\mathrm{~d}, J=7.6 \mathrm{~Hz}, 2 \mathrm{H}, \mathrm{Ar}-\mathrm{H}), 8.10(\mathrm{~d}, J=7.6 \mathrm{~Hz}, 2 \mathrm{H}, \mathrm{Ar}-\mathrm{H})$ and $11.23 \mathrm{ppm}(\mathrm{s}, 1 \mathrm{H}, \mathrm{NH})$; ${ }^{13} \mathrm{C}-\mathrm{NMR}\left(\mathrm{DMSO}-d_{6}\right): \delta 26.03\left(\mathrm{CH}_{2}\right), 115.35(\mathrm{CN}), 118.76,128.64,128.68,129.91,129.97,133.77$, 136.04, 137.22, 138.48, 144.19, 161.40 and $185.82 \mathrm{ppm}$ (Ar-C and CO); MS (EI): m/z (\%) $331\left(\mathrm{M}^{+}\right.$, 74.35), $332\left(\mathrm{M}^{+}+1,16.90\right)$. Anal. calcd. for $\mathrm{C}_{18} \mathrm{H}_{13} \mathrm{~N}_{5} \mathrm{O}_{2}$ (331.34): C, 65.25; H, 3.95; N, 21.14. Found: C, 65.28; H, 4.02; N, 21.20 .

N-(5-Acetyl-2-phenyl-2H-1,2,3-triazol-4-yl)-2-cyanoacetamide (3b). Buff crystals, yield: 95\%, m.p. $205{ }^{\circ} \mathrm{C}$; IR (KBr): $v / \mathrm{cm}^{-1} 3302(\mathrm{NH}), 2262(\mathrm{CN}), 1685,1638$ (2CO); ${ }^{1} \mathrm{H}-\mathrm{NMR}$ (DMSO- $\left.d_{6}\right): \delta$ $2.62\left(\mathrm{~s}, 3 \mathrm{H}, \mathrm{CH}_{3}\right), 4.11\left(\mathrm{~s}, 2 \mathrm{H}, \mathrm{CH}_{2}\right), 7.51(\mathrm{t}, J=8.0 \mathrm{~Hz}, 1 \mathrm{H}, \mathrm{Ar}-\mathrm{H}), 7.62(\mathrm{t}, J=8.0 \mathrm{~Hz}, 2 \mathrm{H}, \mathrm{Ar}-\mathrm{H})$, $8.03(\mathrm{~d}, J=8.0 \mathrm{~Hz}, 2 \mathrm{H}, \mathrm{Ar}-\mathrm{H})$ and $10.67 \mathrm{ppm}(\mathrm{s}, 1 \mathrm{H}, \mathrm{NH}) ;{ }^{13} \mathrm{C}-\mathrm{NMR}\left(\mathrm{DMSO}-d_{6}\right): \delta 26.28\left(\mathrm{CH}_{2}\right)$, $27.71\left(\mathrm{CH}_{3}\right), 115.51(\mathrm{CN}), 118.79,128.85,129.90,137.79,138.46,143.32,161.66$ and $191.69 \mathrm{ppm}$ (Ar-C and CO); MS (EI): m/z (\%) $269\left(\mathrm{M}^{+}, 100\right), 270\left(\mathrm{M}^{+}+1,25.6\right)$. Anal. calcd. for $\mathrm{C}_{13} \mathrm{H}_{11} \mathrm{~N}_{5} \mathrm{O}_{2}$ (269.26): C, 57.99; H, 4.12; N, 26.01. Found: C, 58.04; H, 4.06; N, 25.93.

N-(3-Benzoyl-5-cyano-1-phenyl-1H-pyrazol-4-yl)-2-cyanoacetamide (4). Creamy white crystals, yield: 93\%, m.p. $234{ }^{\circ} \mathrm{C}$; IR (KBr): v/cm ${ }^{-1} 3259(\mathrm{NH}), 2227,2263(2 \mathrm{CN}), 1712,1631$ (2CO); ${ }^{1} \mathrm{H}-\mathrm{NMR}$ $\left(\mathrm{DMSO}-d_{6}, 25^{\circ} \mathrm{C}\right): \delta=4.13\left(\mathrm{~s}, 2 \mathrm{H}, \mathrm{CH}_{2}\right), 7.57-7.72(\mathrm{~m}, 6 \mathrm{H}, \mathrm{Ar}-\mathrm{H}), 7.83(\mathrm{~d}, J=7.6 \mathrm{~Hz}, 2 \mathrm{H}, \mathrm{Ar}-\mathrm{H})$, $8.14(\mathrm{~d}, J=7.6 \mathrm{~Hz}, 2 \mathrm{H}, \mathrm{Ar}-\mathrm{H})$ and $10.83 \mathrm{ppm}(\mathrm{s}, 1 \mathrm{H}, \mathrm{NH}) ;{ }^{13} \mathrm{C}-\mathrm{NMR}\left(\mathrm{DMSO}-d_{6}\right): \delta 25.84\left(\mathrm{CH}_{2}\right)$, $109.64,110.24,115.41,124.20,128.60,128.75,129.76,130.08,130.20,133.74,135.94,137.70$, 141.38, 161.56 and $186.71 \mathrm{ppm}\left(2 \mathrm{CN}, \mathrm{Ar}-\mathrm{C}\right.$ and CO); $\mathrm{MS}(\mathrm{EI}): \mathrm{m} / \mathrm{z}(\%) 355\left(\mathrm{M}^{+}, 73.20\right), 356\left(\mathrm{M}^{+}+1\right.$, 20.35). Anal. calcd. for $\mathrm{C}_{20} \mathrm{H}_{13} \mathrm{~N}_{5} \mathrm{O}_{2}$ (355.36): C, 67.60; H, 3.69; N, 19.71. Found: C, 67.57; H, 3.75; N, 19.74 . 
$N$-(5-Acetyl-3-benzoyl-1-phenyl-1H-pyrazol-4-yl)-2-cyanoacetamide (8). Orange crystals, yield: 93\%, m.p. above $300{ }^{\circ} \mathrm{C}$; IR (KBr): v/cm $3297(\mathrm{NH}), 22114(\mathrm{CN}), 1687$ (br), 1636 (3CO); ${ }^{1} \mathrm{H}-\mathrm{NMR}$ (DMSO- $\left.d_{6}\right): \delta=2.40\left(\mathrm{~s}, 3 \mathrm{H}, \mathrm{CH}_{3}\right), 4.02\left(\mathrm{~s}, 2 \mathrm{H}, \mathrm{CH}_{2}\right), 7.53-7.60(\mathrm{~m}, 7 \mathrm{H}, \mathrm{Ar}-\mathrm{H}), 7.69$ (t, $J=7.2 \mathrm{~Hz}$, $1 \mathrm{H}, \mathrm{Ar}-\mathrm{H}), 8.10(\mathrm{~d}, J=7.6 \mathrm{~Hz}, 2 \mathrm{H}, \mathrm{Ar}-\mathrm{H})$ and $10.45 \mathrm{ppm}(\mathrm{s}, 1 \mathrm{H}, \mathrm{NH}) ;{ }^{13} \mathrm{C}-\mathrm{NMR}$ (DMSO- $\left.d_{6}\right): \delta 25.72$ $\left(\mathrm{CH}_{2}\right), 29.87\left(\mathrm{CH}_{3}\right), 115.54(\mathrm{CN}), 122.13,125.61,128.48,129.13,129.17,130.14,133.42,135.78$, 136.26, 139.65, 142.76, 162.14, 187.20 and $189.62 \mathrm{ppm}$ (Ar-C and CO); MS (EI): m/z (\%) $372\left(\mathrm{M}^{+}\right.$, 30.20), $373\left(\mathrm{M}^{+}+1,8.57\right)$. Anal. calcd. for $\mathrm{C}_{21} \mathrm{H}_{16} \mathrm{~N}_{4} \mathrm{O}_{3}$ (372.39): C, 67.73; H, 4.33; N, 15.05. Found: C, 67.69; H, 4.31; N, 15.10 .

2-Cyano-N-(3,5-diacetyl-1-phenyl-1H-pyrazol-4-yl)acetamide (12). Creamy white crystals, yield: 89\%, m.p. $230{ }^{\circ} \mathrm{C}$; IR (KBr): v/cm $3284(\mathrm{NH}), 2260$ (CN), 1685 (br), 1637 (3CO); ${ }^{1} \mathrm{H}-\mathrm{NMR}$ $\left(\mathrm{DMSO}-d_{6}\right): \delta=2.33\left(\mathrm{~s}, 3 \mathrm{H}, \mathrm{CH}_{3}\right), 2.57\left(\mathrm{~s}, 3 \mathrm{H}, \mathrm{CH}_{3}\right), 4.03\left(\mathrm{~s}, 2 \mathrm{H}, \mathrm{CH}_{2}\right), 7.49-7.57(\mathrm{~m}, 5 \mathrm{H}, \mathrm{Ar}-\mathrm{H})$ and 10.34 ppm (s, 1H, NH); ${ }^{13} \mathrm{C}-\mathrm{NMR}\left(\mathrm{DMSO}-d_{6}\right): \delta 26.16\left(\mathrm{CH}_{2}\right), 27.72\left(\mathrm{CH}_{3}\right), 30.22\left(\mathrm{CH}_{3}\right), 116.01$ (CN), 125.95, 127.94, 129.63, 129.68, 137.18, 140.05, 143.17, 162.64, 190.18 and 193.80 ppm (Ar-C and CO); $\mathrm{MS}(\mathrm{EI}): \mathrm{m} / \mathrm{z}(\%) 310\left(\mathrm{M}^{+}, 55.1\right), 311\left(\mathrm{M}^{+}+1,10.75\right)$. Anal. calcd. for $\mathrm{C}_{16} \mathrm{H}_{14} \mathrm{~N}_{4} \mathrm{O}_{3}(310.31)$ : C, 61.93; H, 4.55; N, 18.05. Found: C, 61.88; H, 4.57; N, 17.98 .

\subsection{General Procedure for the Cyclization of Cyanoacetamides to Azolo Pyridines 5, 6, 10 and 14}

Independent solutions of cyanoacetamides 3, 4, 8 and 12 (5 mmol), in DMF (10 mL) containing anhydrous sodium acetate $(1 \mathrm{~g})$ were stirred at reflux for $1 \mathrm{~h}$. Then, the reaction mixture was cooled to room temperature and poured into ice cold water. The formed crude products were collected by filtration, washed with water and recrystallized from the appropriate solvent to afford the corresponding azolo pyridine derivatives 5, 6, 10 and 14, respectively.

5-Oxo-2,7-diphenyl-4,5-dihydro-2H-[1,2,3]triazolo[4,5-b]pyridine-6-carbonitrile (5a). Recrystallized from a EtOH/dioxane (1:1) mixture as yellow crystals, yield: 87\%, m.p. above $300{ }^{\circ} \mathrm{C}$; IR (KBr): $v / \mathrm{cm}^{-1} 3437(\mathrm{NH}), 2230(\mathrm{CN}), 1651(\mathrm{CO}) ;{ }^{1} \mathrm{H}-\mathrm{NMR}$ (DMSO-d $): \delta 7.47(\mathrm{t}, J=7.6 \mathrm{~Hz}, 1 \mathrm{H}, \mathrm{Ar}-\mathrm{H})$, $7.56(\mathrm{t}, J=8.0 \mathrm{~Hz}, 2 \mathrm{H}, \mathrm{Ar}-\mathrm{H}), 7.65-7.67$ (m, 3H, Ar-H), 7.89 (d, $J=7.6 \mathrm{~Hz}, 2 \mathrm{H}, \mathrm{Ar}-\mathrm{H}), 7.97$ (d, $J=8.0 \mathrm{~Hz}, 2 \mathrm{H}, \mathrm{Ar}-\mathrm{H})$ and $13.30 \mathrm{ppm}(\mathrm{s}, 1 \mathrm{H}, \mathrm{NH}) ;{ }^{13} \mathrm{C}-\mathrm{NMR}\left(\mathrm{DMSO}-d_{6}\right): \delta 105.22,115.73(\mathrm{CN})$, $119.10,128.81,129.21,129.53,129.95,130.53,131.14,131.51,138.62,148.07,151.23$ and 160.14 ppm (Ar-C and CO); MS (EI): m/z (\%) $313\left(\mathrm{M}^{+}, 100\right), 314\left(\mathrm{M}^{+}+1,21.40\right)$. Anal. calcd. for $\mathrm{C}_{18} \mathrm{H}_{11} \mathrm{~N}_{5} \mathrm{O}$ (313.32): C, 69.00; H, 3.54; N, 22.35. Found: C, C, 69.03; H, 3.48; N, 22.39.

\subsubsection{Crystallographic Analysis for 5a}

The crystals were mounted on a glass fiber. All measurements were performed on a Rigaku R-AXIS RAPID diffractometer using filtered Mo-K $\alpha$ radiation. The data were collected at a temperature of $20 \pm 1{ }^{\circ} \mathrm{C}$ to a maximum $2 \theta$ value of $55.0^{\circ}$ using the $\omega$ scanning technique. The structure was solved by charge flipping method and expanded using Fourier techniques. The non-hydrogen atoms were refined anisotropically. Hydrogen atoms were refined using the riding model. 


\subsubsection{Crystal Data}

$\mathrm{C}_{18} \mathrm{H}_{11} \mathrm{~N}_{5} \mathrm{O}, \quad \mathrm{M}=313.32$, triclinic, $\mathrm{a}=6.861(4) \AA, \mathrm{b}=11.229(6) \AA, \mathrm{c}=12.987(7) \AA$, $\mathrm{V}=869.3(8) \AA^{3}, \alpha=110.560(9)^{\circ}, \beta=103.584(9)^{\circ}, \gamma=100.858(9)^{\circ}$, space group: $\mathrm{P}-1, \mathrm{Z}=2$, $\mathrm{D}_{\text {calc }}=1.365 \mathrm{~g} \mathrm{~cm}^{-3}$, No. of reflection measured $3956,2 \theta_{\max }=55.0^{\circ}, \mathrm{R} 1=0.12$. Figure 1 illustrates the structure as determined. Full data can be obtained on request from the CCDC [22].

7-Methyl-5-oxo-2-phenyl-4,5-dihydro-2H-[1,2,3]triazolo[4,5-b]pyridine-6-carbonitrile (5b). Recrystallized from an EtOH/dioxane (2:1) mixture as yellow crystals, yield: $83 \%$, m.p. above $300{ }^{\circ} \mathrm{C}$; IR $(\mathrm{KBr})$ : $v / \mathrm{cm}^{-1} 3410(\mathrm{NH}), 2228(\mathrm{CN}), 1658(\mathrm{CO}) ;{ }^{1} \mathrm{H}-\mathrm{NMR}\left(\mathrm{DMSO}-d_{6}\right.$ ): $\delta=2.64\left(\mathrm{~s}, 3 \mathrm{H}, \mathrm{CH}_{3}\right), 7.48(\mathrm{t}$, $J=8.0 \mathrm{~Hz}, 1 \mathrm{H}, \mathrm{Ar}-\mathrm{H}), 7.58(\mathrm{t}, J=8.0 \mathrm{~Hz}, 2 \mathrm{H}, \mathrm{Ar}-\mathrm{H}), 8.00(\mathrm{~d}, J=8.0 \mathrm{~Hz}, 2 \mathrm{H}, \mathrm{Ar}-\mathrm{H})$, and $13.04 \mathrm{ppm}$ $(\mathrm{s}, 1 \mathrm{H}, \mathrm{NH}) ;{ }^{13} \mathrm{C}-\mathrm{NMR}\left(\mathrm{DMSO}-d_{6}\right): \delta=16.94\left(\mathrm{CH}_{3}\right), 107.37,115.30(\mathrm{CN}), 119.39,129.57,130.41$, 131.96, 139.07, 147.66, 152.55 and $160.07 \mathrm{ppm}$ (Ar-C and CO); MS (EI): m/z (\%) $251\left(\mathrm{M}^{+}, 100\right), 252$ $\left(\mathrm{M}^{+}+1,30.58\right)$. Anal. calcd. for $\mathrm{C}_{13} \mathrm{H}_{9} \mathrm{~N}_{5} \mathrm{O}(251.25)$ : C, 62.15; H, 3.61; N, 27.87. Found: C, 62.19; $\mathrm{H}$, $3.55 ; \mathrm{N}, 27.91$.

5-Oxo-2,7-diphenyl-4,5-dihydro-2H-pyrazolo[4,3-b]pyridine-3,6-dicarbonitrile (6). Recrystallized from a EtOH/dioxane (1:1) mixture as beige crystals, yield: $80 \%$, m.p. above $300{ }^{\circ} \mathrm{C}$; IR $(\mathrm{KBr})$ : $v / \mathrm{cm}^{-1} 3375(\mathrm{NH}), 2227$ (br, 2CN), $1656(\mathrm{CO}) ;{ }^{1} \mathrm{H}-\mathrm{NMR}$ (DMSO- $\left.d_{6}\right): \delta$ 7.64-7.66 (m, 6H, Ar-H), $7.79(\mathrm{~d}, J=7.6 \mathrm{~Hz}, 2 \mathrm{H}, \mathrm{Ar}-\mathrm{H}), 7.85(\mathrm{~d}, J=7.2 \mathrm{~Hz}, 2 \mathrm{H}, \mathrm{Ar}-\mathrm{H})$ and $13.30 \mathrm{ppm}(\mathrm{s}, 1 \mathrm{H}, \mathrm{NH}) ;{ }^{13} \mathrm{C}-\mathrm{NMR}$ (DMSO- $\left.d_{6}\right): \delta=100.37,105.99,109.67,115.83,124.37,129.02,129.91,130.18,130.77,131.22$, 131.71, 133.12, 135.46, 138.11, 152.78 and $159.81 \mathrm{ppm}(2 \mathrm{CN}, \mathrm{Ar}-\mathrm{C}$ and CO); MS (EI): m/z (\%) 337 $\left(\mathrm{M}^{+}, 100\right), 338\left(\mathrm{M}^{+}+1,25.0\right)$. Anal. calcd. for $\mathrm{C}_{20} \mathrm{H}_{11} \mathrm{~N}_{5} \mathrm{O}$ (337.34): C, 71.21; H, 3.29; N, 20.76. Found: 71.19; H, 3.36; N, 20.83 .

3-Benzoyl-7-methyl-5-oxo-1-phenyl-4,5-dihydro-1H-pyrazolo[4,3-b]pyridine-6-carbonitrile (10). Recrystallized from DMSO as brown crystals, yield: $77 \%$, m.p. $238-240{ }^{\circ} \mathrm{C}$; IR (KBr): $v / \mathrm{cm}^{-1} 3381$ $(\mathrm{NH}), 2223(\mathrm{CN}), 1681,1658(2 \mathrm{CO}) ;{ }^{1} \mathrm{H}-\mathrm{NMR}\left(\mathrm{DMSO}-d_{6}\right): \delta 2.12\left(\mathrm{~s}, 3 \mathrm{H}, \mathrm{CH}_{3}\right), 7.56-7.81(\mathrm{~m}, 8 \mathrm{H}$, $\mathrm{Ar}-\mathrm{H}), 8.22(\mathrm{~d}, J=7.2 \mathrm{~Hz}, 2 \mathrm{H}, \mathrm{Ar}-\mathrm{H})$ and $12.02 \mathrm{ppm}(\mathrm{s}, 1 \mathrm{H}, \mathrm{NH}) ;{ }^{13} \mathrm{C}-\mathrm{NMR}$ (DMSO- $\left.d_{6}\right): \delta 18.23$ $\left(\mathrm{CH}_{3}\right), 105.72,115.34(\mathrm{CN}), 126.28,127.04,127.57,128.59,129.15,129.45,129.60,130.07,130.63$, 133.52, 136.00, 138.95, 159.17 and 186.19 ppm (Ar-C and CO); MS (EI): m/z (\%) $354\left(\mathrm{M}^{+}, 100\right), 355$ $\left(\mathrm{M}^{+}+1\right.$, 27.1). Anal. calcd. for $\mathrm{C}_{21} \mathrm{H}_{14} \mathrm{~N}_{4} \mathrm{O}_{2}$ (354.37): C, 71.18; H, 3.95; N, 15.81. Found: 71.24; $\mathrm{H}$, $4.02 ; \mathrm{N}, 15.77$.

\subsubsection{Crystallographic Analysis for $\mathbf{1 0}$}

The crystals were mounted on a glass fiber. All measurements were performed on a Rigaku R-AXIS RAPID diffractometer using filtered Mo-K $\alpha$ radiation. The data were collected at a temperature of $20 \pm 1{ }^{\circ} \mathrm{C}$ to a maximum $2 \theta$ value of $55.0^{\circ}$ using the $\omega$ scanning technique. The structure was solved by charge flipping method and expanded using Fourier techniques. The non-hydrogen atoms were refined anisotropically. Hydrogen atoms were refined using the riding model. 


\subsubsection{Crystal Data}

$\mathrm{C}_{21} \mathrm{H}_{14} \mathrm{~N}_{4} \mathrm{O}_{2}+$ one DMSO molecule, $\mathrm{M}=354.37$, monoclinic, $\mathrm{a}=11.518(2) \AA, \mathrm{b}=9.386(2) \AA$, $\mathrm{c}=19.655(3) \AA, \mathrm{V}=2123.5(5) \AA^{3}, \alpha=\gamma=90.00^{\circ}, \beta=92.069(7)^{\circ}$, space group: $\mathrm{P} 21 / \mathrm{n}, \mathrm{Z}=4$, $\mathrm{D}_{\text {calc }}=1.353 \mathrm{~g} \mathrm{~cm}^{-3}$, No. of reflection measured $4731,2 \theta_{\max }=55.0^{\circ}, \mathrm{R} 1=0.1088$. Figure 2 illustrates the structure as determined. Full data can be obtained on request from the CCDC [23].

3-Acetyl-7-methyl-5-oxo-1-phenyl-4,5-dihydro-1H-pyrazolo[4,3-b]pyridine-6-carbonitrile(14). Recrystallized from DMF as buff crystals, yield: $80 \%$, m.p. above $300{ }^{\circ} \mathrm{C}$; IR (KBr): $v / \mathrm{cm}^{-1} 3183(\mathrm{NH}), 2221(\mathrm{CN})$, 1673, 1639 (2CO); ${ }^{1} \mathrm{H}-\mathrm{NMR}\left(\mathrm{DMSO}-d_{6}\right): \delta 2.15\left(\mathrm{~s}, 3 \mathrm{H}, \mathrm{CH}_{3}\right), 2.60\left(\mathrm{~s}, 3 \mathrm{H}, \mathrm{CH}_{3}\right), 7.49-7.71(\mathrm{~m}, 5 \mathrm{H}$, Ar-H) and $11.91 \mathrm{ppm}(\mathrm{s}, 1 \mathrm{H}, \mathrm{NH}) ;{ }^{13} \mathrm{C}-\mathrm{NMR}(\mathrm{TFA}-d): \delta 19.82\left(\mathrm{CH}_{3}\right), 27.25\left(\mathrm{CH}_{3}\right), 107.63,116.18$ (CN), 125.08, 129.39, 131.18, 131.62, 132.45, 134.48, 138.03, 140.46, 154.60 and 202.41 ppm (Ar-C and CO); MS (EI): m/z (\%) $292\left(\mathrm{M}^{+}, 100\right), 293\left(\mathrm{M}^{+}+1,20.45\right)$. Anal. calcd. for $\mathrm{C}_{16} \mathrm{H}_{12} \mathrm{~N}_{4} \mathrm{O}_{2}(292.30)$ : C, 65.75; H, 4.14; N, 19.17. Found: C, 65.81; H, 4.17; N, 19.19.

\subsection{General Procedure for the Preparation of $\mathbf{1 5}$}

Independent solutions of $p$-nitrophenylacetic acid $(0.9 \mathrm{~g}, 5 \mathrm{mmol})$ in $\mathrm{Ac}_{2} \mathrm{O}(5 \mathrm{~mL})$ were heated in a microwave oven at $100^{\circ} \mathrm{C}$ for $20 \mathrm{~s}$. To these mixtures, 1a,b $(5 \mathrm{mmol})$ were added and the mixtures were heated for further $60 \mathrm{~s}$ at the same temperature. The reaction mixtures were cooled to room temperature the crystalline solid formed were separated by filtration and washed by cold ethanol and then hot ethanol to afford 15a,b, respectively, as pure substances.

N-(5-Benzoyl-2-phenyl-2H-1,2,3-triazol-4-yl)-2-(4-nitrophenyl)acetamide (15a). White crystals, yield: 90\%, m.p. $196{ }^{\circ} \mathrm{C}$; IR (KBr): v/ $\mathrm{cm}^{-1} 3294(\mathrm{NH}), 1690,1632(2 \mathrm{CO}) ;{ }^{1} \mathrm{H}-\mathrm{NMR}\left(\mathrm{CDCl}_{3}\right): \delta 4.02(\mathrm{~s}, 2 \mathrm{H}$, $\left.\mathrm{CH}_{2}\right), 7.43(\mathrm{t}, J=7.6 \mathrm{~Hz}, 1 \mathrm{H}, \mathrm{Ar}-\mathrm{H}), 7.50-7.62(\mathrm{~m}, 6 \mathrm{H}, \mathrm{Ar}-\mathrm{H}), 7.68(\mathrm{t}, J=7.6 \mathrm{~Hz}, 1 \mathrm{H}, \mathrm{Ar}-\mathrm{H}), 8.15(\mathrm{~d}$, $J=8.0 \mathrm{~Hz}, 2 \mathrm{H}, \mathrm{Ar}-\mathrm{H}), 8.27(\mathrm{~d}, J=7.6 \mathrm{~Hz}, 2 \mathrm{H}, \mathrm{Ar}-\mathrm{H}), 8.43$ (d, $J=8.0 \mathrm{~Hz}, 2 \mathrm{H}, \mathrm{Ar}-\mathrm{H})$ and $10.02 \mathrm{ppm}$ $(\mathrm{s}, 1 \mathrm{H}, \mathrm{NH}) ;{ }^{13} \mathrm{C}-\mathrm{NMR}\left(\mathrm{CDCl}_{3}\right): \delta 43.90\left(\mathrm{CH}_{2}\right), 119.47,124.05,128.59,128.76,129.41,130.41$, 130.54, 132.99, 133.95, 135.98, 138.99, 141.04, 147.40, 148.35, 166.42 and $187.60 \mathrm{ppm}$ (Ar-C and $\mathrm{CO})$; $\mathrm{MS}(\mathrm{EI}): \mathrm{m} / \mathrm{z}(\%) 427\left(\mathrm{M}^{+}, 56.75\right), 428\left(\mathrm{M}^{+}+1,15.55\right)$. Anal. calcd. for $\mathrm{C}_{23} \mathrm{H}_{17} \mathrm{~N}_{5} \mathrm{O}_{4}(427.42)$ : C, 64.63; H, 4.01; N, 16.39. Found: 64.59; H, 3.94; N, 16.43.

N-(5-Acetyl-2-phenyl-2H-1,2,3-triazol-4-yl)-2-(4-nitrophenyl)acetamide (15b). Beige crystals, yield: 87\%, m.p. $207{ }^{\circ} \mathrm{C}$; IR (KBr): v/cm $3358(\mathrm{NH}), 1686,1641(2 \mathrm{CO}) ;{ }^{1} \mathrm{H}-\mathrm{NMR}\left(\mathrm{CDCl}_{3}\right): \delta 2.69$ (s, 3H, $\left.\mathrm{CH}_{3}\right), 3.99$ (s, 2H, $\left.\mathrm{CH}_{2}\right), 7.43$ (t, $\left.J=7.6 \mathrm{~Hz}, 1 \mathrm{H}, \mathrm{Ar}-\mathrm{H}\right), 7.51(\mathrm{t}, J=7.6 \mathrm{~Hz}, 2 \mathrm{H}, \mathrm{Ar}-\mathrm{H}), 7.60$ (d, $J=8.0 \mathrm{~Hz}, 2 \mathrm{H}, \mathrm{Ar}-\mathrm{H}), 8.12$ (d, $J=7.6 \mathrm{~Hz}, 2 \mathrm{H}, \mathrm{Ar}-\mathrm{H}), 8.26$ (d, $J=8.0 \mathrm{~Hz}, 2 \mathrm{H}, \mathrm{Ar}-\mathrm{H})$ and $9.51 \mathrm{ppm}$ (s, $1 \mathrm{H}, \mathrm{NH}) ;{ }^{13} \mathrm{C}-\mathrm{NMR}\left(\mathrm{CDCl}_{3}\right): \delta 27.02\left(\mathrm{CH}_{3}\right), 43.81\left(\mathrm{CH}_{2}\right), 119.38,124.06,128.74,129.42,130.56$, 133.67, 138.99, 140.97, 146.14, 147.43, 166.43 and $195.23 \mathrm{ppm}$ (Ar-C and CO); MS (EI): m/z (\%) $365\left(\mathrm{M}^{+}, 27.35\right), 366\left(\mathrm{M}^{+}+1,6.91\right)$. Anal. calcd. for $\mathrm{C}_{18} \mathrm{H}_{15} \mathrm{~N}_{5} \mathrm{O}_{4}$ (365.35): C, 59.18; H, 4.14; N, 19.17 . Found: C, 59.24; H, 4.07; N, 19.14. 


\subsection{General Procedure for the Preparation of $\mathbf{1 6}$}

Independent solutions of amides 15a,b $(5 \mathrm{mmol})$ in DMF $(10 \mathrm{~mL})$ containing anhydrous sodium acetate $(1 \mathrm{~g})$ were stirred at reflux for $1 \mathrm{~h}$. Then, the reaction mixtures were cooled to $\mathrm{rt}$ and poured onto ice cold water. The crude products were collected by filtration, washed with water and recrystallized from the appropriate solvent to afford the corresponding azolo pyridine derivatives 16a,b respectively.

6-(4-Nitrophenyl)-2,7-diphenyl-2,4-dihydro[1,2,3]triazolo[4,5-b]pyridin-5-one (16a). Recrystallized from an EtOH/dioxane (1:1) mixture as yellow crystals, yield: $82 \%$, m.p. $302{ }^{\circ} \mathrm{C}$; IR $(\mathrm{KBr}): v / \mathrm{cm}^{-1}$ $3435(\mathrm{NH}), 1645$ (CO); ${ }^{1} \mathrm{H}-\mathrm{NMR}$ (DMSO- $\left.d_{6}\right): \delta 7.32-7.35(\mathrm{~m}, 5 \mathrm{H}, \mathrm{Ar}-\mathrm{H}), 7.44$ (d, $J=8.0 \mathrm{~Hz}, 3 \mathrm{H}$, Ar-H), 7.56 (t, $J=7.6 \mathrm{~Hz}, 2 \mathrm{H}, \mathrm{Ar}-\mathrm{H}), 8.00$ (d, $J=8.0 \mathrm{~Hz}, 2 \mathrm{H}, \mathrm{Ar}-\mathrm{H}), 8.10$ (d, $J=8.0 \mathrm{~Hz}, 2 \mathrm{H}, \mathrm{Ar}-\mathrm{H})$ and $12.90 \mathrm{ppm}(\mathrm{s}, 1 \mathrm{H}, \mathrm{NH}) ;{ }^{13} \mathrm{C}-\mathrm{NMR}\left(\mathrm{DMSO}-d_{6}\right): \delta 118.62,122.51,128.18,128.25,128.89,129.82$, $129.86,130.66,132.29,132.64,133.14,139.02,140.68,142.80,146.36,146.65$ and $161.83 \mathrm{ppm}$ (Ar-C and CO); MS (EI): m/z (\%) $409\left(\mathrm{M}^{+}, 100\right), 410\left(\mathrm{M}^{+}+1,30.72\right)$. Anal. calcd. for $\mathrm{C}_{23} \mathrm{H}_{15} \mathrm{~N}_{5} \mathrm{O}_{3}$ (409.41): C, 67.48; H, 3.69; N, 17.11. Found: C, 67.51; H, 3.70; N, 17.08.

7-Methyl-6-(4-Nitrophenyl)-2-phenyl-2,4-dihydro[1,2,3]triazolo[4,5-b]-pyridin-5-one (16b). Recrystallized from an EtOH/dioxane (2:1) mixture as buff crystals, yield: $85 \%$, m.p. $298^{\circ} \mathrm{C}$; IR (KBr): $v / \mathrm{cm}^{-1}$ $3285(\mathrm{NH}), 1643(\mathrm{CO}) ;{ }^{1} \mathrm{H}-\mathrm{NMR}\left(\mathrm{DMSO}-d_{6}\right): \delta=2.30\left(\mathrm{~s}, 3 \mathrm{H}, \mathrm{CH}_{3}\right), 7.46(\mathrm{t}, J=7.6 \mathrm{~Hz}, 1 \mathrm{H}, \mathrm{Ar}-\mathrm{H})$, $7.58-7.63(\mathrm{~m}, 4 \mathrm{H}, \mathrm{Ar}-\mathrm{H}), 8.05(\mathrm{~d}, J=8.0 \mathrm{~Hz}, 2 \mathrm{H}, \mathrm{Ar}-\mathrm{H}), 8.30(\mathrm{~d}, J=8.0 \mathrm{~Hz}, 2 \mathrm{H}, \mathrm{Ar}-\mathrm{H})$, and $12.67 \mathrm{ppm}(\mathrm{s}, 1 \mathrm{H}, \mathrm{NH}) ;{ }^{13} \mathrm{C}-\mathrm{NMR}\left(\mathrm{DMSO}-d_{6}\right): \delta 15.16\left(\mathrm{CH}_{3}\right), 118.48,122.99,128.13,129.84,131.14$, 131.86, 133.08, 138.08, 139.10, 142.38, 145.94, 146.81 and $161.63 \mathrm{ppm}$ (Ar-C and CO); MS (EI): m/z (\%) $347\left(\mathrm{M}^{+}, 100\right), 348\left(\mathrm{M}^{+}+1,21.87\right)$. Anal. calcd. for $\mathrm{C}_{18} \mathrm{H}_{13} \mathrm{~N}_{5} \mathrm{O}_{3}$ (347.34): C, 62.25; H, 3.77; N, 20.16. Found: C, 62.19; H, 3.81; N, 20.12.

\subsection{General Procedure for the Preparation of 20a-f}

Independent mixtures of 5-amino-1,2,3-triazoles 1a,b $(10 \mathrm{mmol})$, active methylene compounds 17a-c $(15 \mathrm{mmol})$ and zeolite $\left(10 \%\right.$ by weight) were heated at $150{ }^{\circ} \mathrm{C}$ for $1 \mathrm{~h}$. Dioxane was added to the reaction mixtures followed by filtration to remove the zeolite. The crystals formed upon cooling the filtrates were collected by filtration and washed with methanol.

Ethyl-5-oxo-2,7-diphenyl-4,5-dihydro-2H-[1,2,3]triazolo[4,5-b]pyridine-6-carboxylate (20a). Pale orange crystals, yield: $76 \%$, m.p. $205^{\circ} \mathrm{C}$; IR (KBr): v/cm ${ }^{-1} 3391(\mathrm{NH}), 1733,1650$ (2CO); ${ }^{1} \mathrm{H}-\mathrm{NMR}$ $\left(\mathrm{DMSO}-d_{6}\right): \delta 1.30\left(\mathrm{t}, J=7.2 \mathrm{~Hz}, 3 \mathrm{H}, \mathrm{CH}_{3} \mathrm{CH}_{2}\right), 4.13\left(\mathrm{q}, J=7.2 \mathrm{~Hz}, 2 \mathrm{H}, \mathrm{CH}_{3} C_{2}\right), 7.46(\mathrm{t}, J=7.6 \mathrm{~Hz}$, 1H, Ar-H), 7.56-7.60 (m, 5H, Ar-H), 7.65-7.67 (m, 2H, Ar-H), $8.00(\mathrm{~d}, J=7.6 \mathrm{~Hz}, 2 \mathrm{H}, \mathrm{Ar}-\mathrm{H})$ and $13.00 \mathrm{ppm}(\mathrm{s}, 1 \mathrm{H}, \mathrm{NH}) ;{ }^{13} \mathrm{C}-\mathrm{NMR}\left(\mathrm{DMSO}-d_{6}\right): \delta 13.65\left(\mathrm{CH}_{3} \mathrm{CH}_{2}\right), 61.17\left(\mathrm{CH}_{3} \mathrm{CH}_{2}\right), 118.81,128.54$, $128.57,128.67,129.86,129.87,130.10,130.85,132.11,138.97,140.27,146.93,160.14$ and 165.01 ppm (Ar-C and CO); MS (EI): m/z (\%) $360\left(\mathrm{M}^{+}, 100\right), 361\left(\mathrm{M}^{+}+1,24.55\right)$. Anal. Calcd. for $\mathrm{C}_{20} \mathrm{H}_{16} \mathrm{~N}_{4} \mathrm{O}_{3}$ (360.38): C, 66.66; H, 4.48; N, 15.55. Found: C, 66.72; H, 4.40; N, 15.49.

6-Benzoyl-2,7-diphenyl-2,4-dihydro[1,2,3]triazolo[4,5-b]pyridin-5-one (20b). Canary yellow crystals, yield: $80 \%$, m.p. above $300{ }^{\circ} \mathrm{C}$; IR (KBr): $v / \mathrm{cm}^{-1} 3423(\mathrm{NH}), 1672,1644$ (2CO); ${ }^{1} \mathrm{H}-\mathrm{NMR}$ (DMSO- 
$\left.d_{6}\right): \delta$ 7.39-7.41 (m, 3H, Ar-H), $7.46(\mathrm{t}, J=8.0 \mathrm{~Hz}, 3 \mathrm{H}, \mathrm{Ar}-\mathrm{H}), 7.52-7.62(\mathrm{~m}, 5 \mathrm{H}, \mathrm{Ar}-\mathrm{H}), 7.89(\mathrm{~d}$, $J=8.0 \mathrm{~Hz}, 2 \mathrm{H}, \mathrm{Ar}-\mathrm{H}), 8.02(\mathrm{~d}, J=8.0 \mathrm{~Hz}, 2 \mathrm{H}, \mathrm{Ar}-\mathrm{H})$ and $12.99 \mathrm{ppm}(\mathrm{s}, 1 \mathrm{H}, \mathrm{NH}) ;{ }^{13} \mathrm{C}-\mathrm{NMR}$ (DMSO$\left.d_{6}\right): \delta=118.79,128.14,128.45,128.88,129.01,129.73,129.89,131.40,131.67,132.08,133.17$, 133.90, 136.41, 139.06, 140.49, 147.13, 161.25 and $193.91 \mathrm{ppm}$ (Ar-C and CO); MS (EI): m/z (\%) $392\left(\mathrm{M}^{+}, 100\right), 393\left(\mathrm{M}^{+}+1,27.84\right)$. Anal. calcd. for $\mathrm{C}_{24} \mathrm{H}_{16} \mathrm{~N}_{4} \mathrm{O}_{2}$ (392.42): C, 73.46; H, 4.11; N, 14.28 . Found: C, 73.52; H, 4.08; N, 14.36.

6-Acetyl-2,7-diphenyl-2,4-dihydro[1,2,3]triazolo[4,5-b]pyridin-5-one (20c). Pale yellow crystals, yield: 79\%, m.p. $249{ }^{\circ} \mathrm{C}$; IR (KBr): $v / \mathrm{cm}^{-1} 3299(\mathrm{NH}), 1709,1646$ (2CO); ${ }^{1} \mathrm{H}-\mathrm{NMR}$ (DMSO- $\left.d_{6}\right): \delta$ $2.33\left(\mathrm{~s}, 3 \mathrm{H}, \mathrm{CH}_{3}\right), 7.47$ (t, $\left.J=7.6 \mathrm{~Hz}, 1 \mathrm{H}, \mathrm{Ar}-\mathrm{H}\right), 7.53-7.60(\mathrm{~m}, 7 \mathrm{H}, \mathrm{Ar}-\mathrm{H}), 7.99$ (d, J= 7.6 Hz, 2H, $\mathrm{Ar}-\mathrm{H})$ and $12.96 \mathrm{ppm}(\mathrm{s}, 1 \mathrm{H}, \mathrm{NH}) ;{ }^{13} \mathrm{C}-\mathrm{NMR}$ (DMSO- $\left.d_{6}\right): \delta 31.57\left(\mathrm{CH}_{3}\right), 118.72,128.43,128.60$, 128.96, 129.76, 129.80, 131.38, 132.13, 133.69, 138.95, 139.20, 146.64, 160.80 and $201.47 \mathrm{ppm}$ (Ar-C and CO); MS (EI): m/z (\%) $330\left(\mathrm{M}^{+}, 100\right), 331\left(\mathrm{M}^{+}+1,19.84\right)$. Anal. calcd. for $\mathrm{C}_{19} \mathrm{H}_{14} \mathrm{~N}_{4} \mathrm{O}_{2}$ (330.35): C, 69.08; H, 4.27; N, 16.96. Found: C, 68.98; H, 4.35; N, 16.88.

Ethyl-7-methyl-5-oxo-2-phenyl-4,5-dihydro-2H-[1,2,3]triazolo[4,5-b]pyridine-6-carboxylate (20d). Yellow crystals, yield: $82 \%$, m.p. $219^{\circ} \mathrm{C}$; IR (KBr): $v / \mathrm{cm}^{-1} 3263(\mathrm{NH}), 1735,1659$ (2CO); ${ }^{1} \mathrm{H}-\mathrm{NMR}$ (DMSO- $\left.d_{6}\right): \delta 1.31\left(\mathrm{t}, J=7.2 \mathrm{~Hz}, 3 \mathrm{H}, \mathrm{CH}_{3} \mathrm{CH}_{2}\right), 2.44\left(\mathrm{~s}, 3 \mathrm{H}, \mathrm{CH}_{3}\right), 4.33$ (q, J=7.2 Hz, 2H, $\mathrm{CH}_{3} \mathrm{CH}_{2}$ ), $7.47(\mathrm{t}, J=8.0 \mathrm{~Hz}, 1 \mathrm{H}, \mathrm{Ar}-\mathrm{H}), 7.60(\mathrm{t}, J=8.0 \mathrm{~Hz}, 2 \mathrm{H}, \mathrm{Ar}-\mathrm{H}), 8.04(\mathrm{~d}, J=8.0 \mathrm{~Hz}, 2 \mathrm{H}, \mathrm{Ar}-\mathrm{H})$ and 12.74 ppm $(\mathrm{s}, 1 \mathrm{H}, \mathrm{NH}) ;{ }^{13} \mathrm{C}-\mathrm{NMR}\left(\mathrm{DMSO}-d_{6}\right): \delta 14.04\left(\mathrm{CH}_{3} \mathrm{CH}_{2}\right), 14.31\left(\mathrm{CH}_{3}\right), 61.28\left(\mathrm{CH}_{3} \mathrm{CH}_{2}\right), 118.52$, 127.22, 128.33, 129.61, 129.76, 131.86, 138.90, 146.12, 159.82 and 165.08 ppm (Ar-C and CO); MS (EI): $\mathrm{m} / \mathrm{z}(\%) 298\left(\mathrm{M}^{+}, 61.90\right), 299\left(\mathrm{M}^{+}+1,12.75\right)$. Anal. calcd. for $\mathrm{C}_{15} \mathrm{H}_{14} \mathrm{~N}_{4} \mathrm{O}_{3}$ (298.30): C, 60.40; $\mathrm{H}$, 4.73; N, 18.78. Found: C, 60.33; H, 4.84; N, 18.76 .

6-Benzoyl-7-methyl-2-phenyl-2,4-dihydro[1,2,3]triazolo[4,5-b]pyridin-5-one (20e). Creamy white crystals, yield: $79 \%$, m.p. $298{ }^{\circ} \mathrm{C}$; IR (KBr): v/ $\mathrm{cm}^{-1} 3429$ (NH), 1668, 1641 (2CO); ${ }^{1} \mathrm{H}-\mathrm{NMR}$ (DMSO$\left.d_{6}\right): \delta 2.30\left(\mathrm{~s}, 3 \mathrm{H}, \mathrm{CH}_{3}\right), 7.47(\mathrm{t}, J=7.6 \mathrm{~Hz}, 1 \mathrm{H}, \mathrm{Ar}-\mathrm{H}), 7.55(\mathrm{t}, J=8.0 \mathrm{~Hz}, 2 \mathrm{H}, \mathrm{Ar}-\mathrm{H}), 7.61(\mathrm{t}$, $J=7.6 \mathrm{~Hz}, 2 \mathrm{H}, \mathrm{Ar}-\mathrm{H}), 7.69(\mathrm{t}, J=7.6 \mathrm{~Hz}, 1 \mathrm{H}, \mathrm{Ar}-\mathrm{H}), 7.92$ (d, $J=7.6 \mathrm{~Hz}, 2 \mathrm{H}, \mathrm{Ar}-\mathrm{H}), 8.06$ (d, $J=8.0 \mathrm{~Hz}, 2 \mathrm{H}, \mathrm{Ar}-\mathrm{H})$ and $12.75 \mathrm{ppm}(\mathrm{s}, 1 \mathrm{H}, \mathrm{NH}) ;{ }^{13} \mathrm{C}-\mathrm{NMR}\left(\mathrm{DMSO}-d_{6}\right): \delta 14.18\left(\mathrm{CH}_{3}\right), 118.62$, 128.34, 129.01, 129.04, 129.89, 131.90, 132.57, 134.12, 136.24, 138.83, 139.08, 146.45, 161.04 and $194.41 \mathrm{ppm}$ (Ar-C and CO); MS (EI): m/z (\%) $330\left(\mathrm{M}^{+}, 100\right), 331\left(\mathrm{M}^{+}+1,22.88\right)$. Anal. calcd. for $\mathrm{C}_{19} \mathrm{H}_{14} \mathrm{~N}_{4} \mathrm{O}_{2}$ (330.35): C, 69.08; H, 4.27; N, 16.96. Found: C, 69.15; H, 4.21; N, 17.02.

6-Acetyl-7-methyl-2-phenyl-2,4-dihydro[1,2,3]triazolo[4,5-b]pyridin-5-one (20f). Yellow crystals, yield: 86\%, m.p. $242{ }^{\circ} \mathrm{C}$; IR (KBr): $v / \mathrm{cm}^{-1} 3435(\mathrm{NH}), 1694,1648$ (2CO); ${ }^{1} \mathrm{H}-\mathrm{NMR}$ (DMSO- $\left.d_{6}\right): \delta$ $2.36\left(\mathrm{~s}, 3 \mathrm{H}, \mathrm{CH}_{3}\right), 2.47\left(\mathrm{~s}, 3 \mathrm{H}, \mathrm{CH}_{3}\right), 7.44(\mathrm{t}, J=8.0 \mathrm{~Hz}, 1 \mathrm{H}, \mathrm{Ar}-\mathrm{H}), 7.57$ (t, $\left.J=8.0 \mathrm{~Hz}, 2 \mathrm{H}, \mathrm{Ar}-\mathrm{H}\right)$, $8.00(\mathrm{~d}, J=8.0 \mathrm{~Hz}, 2 \mathrm{H}, \mathrm{Ar}-\mathrm{H})$ and $12.71 \mathrm{ppm}(\mathrm{s}, 1 \mathrm{H}, \mathrm{NH}) ;{ }^{13} \mathrm{C}-\mathrm{NMR}\left(\mathrm{DMSO}-d_{6}\right): \delta 13.98\left(\mathrm{CH}_{3}\right)$, $30.98\left(\mathrm{CH}_{3}\right), 118.57,128.34,129.79,132.46,133.31,138.53,138.95,146.09,160.99$ and $201.93 \mathrm{ppm}$ (Ar-C and CO); MS (EI): m/z (\%) $268\left(\mathrm{M}^{+}, 76.45\right), 269\left(\mathrm{M}^{+}+1,13.89\right)$. Anal. calcd. for $\mathrm{C}_{14} \mathrm{H}_{12} \mathrm{~N}_{4} \mathrm{O}_{2}$ (268.28): C, 62.68; H, 4.51; N, 20.88. Found: C, 62.74; H, 4.47; N, 20.94. 


\subsubsection{Crystallographic Analysis for $20 f$}

The crystals were mounted on a glass fiber. All measurements were performed on a Rigaku R-AXIS RAPID diffractometer using filtered Mo-K $\alpha$ radiation. The data were collected at a temperature of $20 \pm 1{ }^{\circ} \mathrm{C}$ to a maximum $2 \theta$ value of $55.0^{\circ}$ using the $\omega$ scanning technique. The structure was solved by charge flipping method and expanded using Fourier techniques. The non-hydrogen atoms were refined anisotropically. Hydrogen atoms were refined using the riding model.

\subsubsection{Crystal Data}

$\mathrm{C}_{14} \mathrm{H}_{12} \mathrm{~N}_{4} \mathrm{O}_{2}, \mathrm{M}=268.28$, triclinic, $\mathrm{a}=4.003(4) \AA, \mathrm{b}=13.07(2) \AA, \mathrm{c}=13.49(2) \AA, \mathrm{V}=643(1) \AA^{3}, \alpha=$ $113.65(1)^{\circ}, \beta=91.13(2)^{\circ}, \gamma=94.89(2)^{\circ}$, space group: $\mathrm{P}-1, \mathrm{Z}=2, D_{\text {calc }}=1.385 \mathrm{~g} \mathrm{~cm}^{-3}$, No. of reflection measured $2935,2 \theta_{\max }=55.0^{\circ}, \mathrm{R} 1=0.053$. Figure 3 illustrates the structure as determined. Full data can be obtained on request from the CCDC [24].

\subsection{N-(5-Benzoyl-2-phenyl--2H-1,2,3-triazol-4-yl)-5-oxo-2,7-diphenyl-4,5-dihydro-2H-[1,2,3]-} triazolo[4,5-b]pyridine-6-carboxamide (22)

A mixture of 5-amino-1,2,3-triazole 1a $(0.66 \mathrm{~g}, 2.5 \mathrm{mmol})$, pyrazolo[4,3-b]pyridine 20a $(0.9 \mathrm{~g}$, $2.5 \mathrm{mmol})$ and zeolite $(10 \%$ by weight $)$ in dioxane $(10 \mathrm{~mL})$ was stirred at reflux for $2 \mathrm{~h}$, filtered to remove the zeolite, and cooled to room temperature. The solid which formed was collected by filtration, washed with ethanol, and recrystallized from dioxane giving beige crystals, yield: $68 \%$, m.p. $271{ }^{\circ} \mathrm{C}$; IR (KBr): $v / \mathrm{cm}^{-1} 3404,3314(2 \mathrm{NH}), 1711,1663,1635$ (3CO); ${ }^{1} \mathrm{H}-\mathrm{NMR}$ (DMSO- $d_{6}$ ): $\delta=7.49-7.73(\mathrm{~m}, 14 \mathrm{H}, \mathrm{Ar}-\mathrm{H}), 8.01-8.12(\mathrm{~m}, 6 \mathrm{H}, \mathrm{Ar}-\mathrm{H}), 11.48(\mathrm{~s}, 1 \mathrm{H}, \mathrm{NH})$ and $12.93 \mathrm{ppm}(\mathrm{s}, 1 \mathrm{H}$, $\mathrm{NH}) ;{ }^{13} \mathrm{C}-\mathrm{NMR}\left(\mathrm{DMSO}-d_{6}\right): \delta 118.83,128.52,128.60,128.70,129.04,129.75,129.90,130.87$, $131.39,132.26,133.67,136.35,136.46,138.61,139.01,140.66,144.50,147.02,161.14,162.51$, and $185.99 \mathrm{ppm}$ (Ar-C and CO); MS (EI): m/z (\%) $578\left(\mathrm{M}^{+}, 46.25\right), 579\left(\mathrm{M}^{+}+1,17.30\right)$. Anal. calcd. for $\mathrm{C}_{33} \mathrm{H}_{22} \mathrm{~N}_{8} \mathrm{O}_{3}$ (578.60): C, 68.51; H, 3.83; N, 19.37. Found: C, 68.44; H, 3.87; N, 19.42.

\section{8. (E)-Ethyl-3-(5-acetyl-2-phenyl-2H-1,2,3-triazol-4-ylamino)but-2-enoate (23)}

A mixture of 5-amino-1,2,3-triazole $\mathbf{1 b}(2.02 \mathrm{~g}, 10 \mathrm{mmol})$ and ethyl acetoacetate $(1.95 \mathrm{~g}, 15 \mathrm{mmol})$ was fused at $140{ }^{\circ} \mathrm{C}$ for $20 \mathrm{~min}$. The mixture was poured into water and cooled to room temperature. The crude solid which formed was collected by filtration, washed with cold ethanol, and recrystallized from ethanol to give creamy white crystals, yield: $71 \%$, m.p. $158{ }^{\circ} \mathrm{C}$; IR $(\mathrm{KBr}): v / \mathrm{cm}^{-1} 3435(\mathrm{NH})$, 1672, 1620 (2CO); ${ }^{1} \mathrm{H}-\mathrm{NMR}\left(\mathrm{CDCl}_{3}\right): \delta 1.30$ (t, $\left.J=7.2 \mathrm{~Hz}, 3 \mathrm{H}, \mathrm{CH}_{3} \mathrm{CH}_{2}\right), 2.50\left(\mathrm{~s}, 3 \mathrm{H}, \mathrm{CH}_{3}\right), 2.68$ (s, $\left.3 \mathrm{H}, \mathrm{CH}_{3} \mathrm{CO}\right), 4.27$ (q, $\left.J=7.2 \mathrm{~Hz}, 2 \mathrm{H}, \mathrm{CH}_{3} \mathrm{CH}_{2}\right), 4.93(\mathrm{~s}, 1 \mathrm{H}$, olefinic $\mathrm{CH}), 7.38(\mathrm{t}, J=8.0 \mathrm{~Hz}, 1 \mathrm{H}$, Ar-H), 7.49 (t, $J=8.0 \mathrm{~Hz}, 2 \mathrm{H}, \mathrm{Ar}-\mathrm{H}), 8.05$ (d, $J=8.0 \mathrm{~Hz}, 2 \mathrm{H}, \mathrm{Ar}-\mathrm{H})$ and $11.95 \mathrm{ppm}(\mathrm{s}, 1 \mathrm{H}, \mathrm{NH})$; ${ }^{13} \mathrm{C}-\mathrm{NMR}\left(\mathrm{CDCl}_{3}\right): \delta 14.55\left(\mathrm{CH}_{3} \mathrm{CH}_{2}\right), 22.75\left(\mathrm{CH}_{3}\right), 26.84\left(\mathrm{CH}_{3}\right), 59.40\left(\mathrm{CH}_{3} \mathrm{CH}_{2}\right), 92.61,118.76$, $128.05,129.35,133.81,139.16,149.00,154.87,168.94$ and $193.51 \mathrm{ppm}$ (Ar-C, olefinic $\mathrm{C}$ and $\mathrm{CO}$ ); MS (EI): $\mathrm{m} / \mathrm{z}(\%) 314\left(\mathrm{M}^{+}, 100\right), 315\left(\mathrm{M}^{+}+1,22.85\right)$. Anal. Calcd. for $\mathrm{C}_{16} \mathrm{H}_{18} \mathrm{~N}_{4} \mathrm{O}_{3}$ (314.35): C, 61.14; H, 5.77; N, 17.82. Found: C, 61.17; H, 5.75; N, 17.86 . 


\subsubsection{Crystallographic Analysis for 23}

The crystals were mounted on a glass fiber. All measurements were performed on a Rigaku R-AXIS RAPID diffractometer using filtered Mo-K $\alpha$ radiation. The data were collected at a temperature of $20 \pm 1{ }^{\circ} \mathrm{C}$ to a maximum $2 \theta$ value of $55.0^{\circ}$ using the $\omega$ scanning technique. The structure was solved by charge flipping method and expanded using Fourier techniques. The non-hydrogen atoms were refined anisotropically. Hydrogen atoms were refined using the riding model.

\subsubsection{Crystal Data}

$\mathrm{C}_{16} \mathrm{H}_{18} \mathrm{~N}_{4} \mathrm{O}_{3}, \quad \mathrm{M}=314.35$, monoclinic, $\mathrm{a}=4.949(2) \AA, \mathrm{b}=14.130(4) \AA, \mathrm{c}=23.315(7) \AA$, $\mathrm{V}=1629.14(9) \AA^{3}, \alpha=\gamma=90.00^{\circ}, \beta=92.190(2)^{\circ}$, space group: $\mathrm{P} 2_{1} / \mathrm{n}, Z=4, \mathrm{D}_{\text {calc }}=1.282 \mathrm{~g} \mathrm{~cm}^{-3}$, No. of reflection measured $3733,2 \theta_{\max }=55.0^{\circ}, \mathrm{R} 1=0.0807$. Figure 4 illustrates the structure as determined. Full data can be obtained on request from the CCDC [25].

\subsection{Ethyl-5,7-dimethyl-2-phenyl-2H-[1,2,3]triazolo[4,5-b]pyridine-6-carboxylate (24)}

A solution of $23(1.57 \mathrm{~g}, 5 \mathrm{mmol})$ in DMF $(10 \mathrm{~mL})$ containing anhydrous sodium acetate $(1 \mathrm{~g})$ was stirred at reflux for $1 \mathrm{~h}$. The mixture was cooled to room temperature and poured into ice cold water. The formed solid was collected by filtration, washed with water and recrystallized from $\mathrm{EtOH} / \mathrm{H}_{2} \mathrm{O}$ (2:1) to give pale brown crystals, yield: $71 \%$, m.p. $78{ }^{\circ} \mathrm{C}$; IR (KBr): v/cm ${ }^{-1} 1719$ (CO); ${ }^{1} \mathrm{H}-\mathrm{NMR}$ $\left(\mathrm{DMSO}-d_{6}\right): \delta 1.46\left(\mathrm{t}, J=7.2 \mathrm{~Hz}, 3 \mathrm{H}, \mathrm{CH}_{3} \mathrm{CH}_{2}\right), 2.74\left(\mathrm{~s}, 3 \mathrm{H}, \mathrm{CH}_{3}\right), 2.75\left(\mathrm{~s}, 3 \mathrm{H}, \mathrm{CH}_{3}\right), 4.50$ (q, $\left.J=7.2 \mathrm{~Hz}, 2 \mathrm{H}, \mathrm{CH}_{3} \mathrm{CH}_{2}\right), 7.49(\mathrm{t}, J=8.0 \mathrm{~Hz}, 1 \mathrm{H}, \mathrm{Ar}-\mathrm{H}), 7.57(\mathrm{t}, J=8.0 \mathrm{~Hz}, 2 \mathrm{H}, \mathrm{Ar}-\mathrm{H})$ and $8.40 \mathrm{ppm}$ $(\mathrm{d}, J=8.0 \mathrm{~Hz}, 2 \mathrm{H}, \mathrm{Ar}-\mathrm{H}) ;{ }^{13} \mathrm{C}-\mathrm{NMR}\left(\mathrm{DMSO}-d_{6}\right): \delta 14.27\left(\mathrm{CH}_{3}\right), 14.71\left(\mathrm{CH}_{3}\right), 24.42\left(\mathrm{CH}_{3}\right), 61.89$ $\left(\mathrm{CH}_{2}\right), 120.59,128.72,129.50,129.55,136.67,137.78,140.14,154.96,158.88$ and $168.10 \mathrm{pm}$ (Ar-C and CO); MS (EI): m/z (\%) $296\left(\mathrm{M}^{+}, 100\right), 297\left(\mathrm{M}^{+}+1,29.8\right)$. Anal. calcd. for $\mathrm{C}_{16} \mathrm{H}_{16} \mathrm{~N}_{4} \mathrm{O}_{2}(296.33)$ : C, 64.85; H, 5.44; N, 18.91. Found: C, 64.78; H, 5.51; N, 18.94.

\section{Conclusions}

A simple and efficient approach to the preparation of condensed pyridines, utilizing $o$-acyl heteroarmatic amines as precursors, has been developed. By using the new approach, difficulties with the classical synthesis of these substances from active methylene compounds and $o$-aminonitriles are overcome.

\section{Acknowledgments}

Support of this work was provided by the University of Kuwait through a research grant (SC03/07). The facilities of Analab/SAF supported by research grants GS01/01, GS01/05, GS01/03 and GS03/08 are gratefully acknowledged. 


\section{References and Notes}

1. Straub, A.; Stasch, J.-P.; Alonso-Alija, C.; Benet-Buchholz, J.; Ducke, B.; Feurer, A.; Fürstner, C. NO-Independent stimulators of soluble guanylate cyclase. Bioorg. Med. Chem. Lett. 2001, 11, 781-784.

2. Tono-Oka, S.; Azuma, I. Triazolo[4,5-b]- and Imidazo[4,5-b]pyridines as Substrates of NAD Glycohydrolase: Evidence for Two Competitive Transglycosylatin Reaction Sites. Liebigs Ann. 1996, 1996, 863-865.

3. Gaston, M.A.; Dias, L.R.; Freitas, A.C.; Miranda, A.I.P.; Barrerio, E.J. Synthesis and analgesic properties of new 4-arylhydrazone 1-H pyrazole[3,4-b]pyridine derivatives. Pharm. Acta Helv. 1996, 71, 213-219.

4. Holt, J.; Fiksdahl, A. N-Acyl and N-Alkoxycarbonyl Derivatives of 1-H-1,2,3-Triazolo[4,5-c]pyridine; Preparation and Application. J. Heterocycl. Chem. 2006, 43, 417-423.

5. Behbehani, H.; Ibrahim, H.M.; Makhseed, S.; Mahmoud, H. Applications of 2-arylhydrazononitriles in synthesis: Preparation of new indole containing 1,2,3-triazole, pyrazole and pyrazolo[1,5-a]pyrimidine derivatives and evaluation of their antimicrobial activities. Eur. J. Med. Chem. 2011, 46, 1813-1820.

6. Poreba, K.; Opolski, A.; Wietrzyk, J. Synthesis and antiproliferative activity in vitro of new 3-substituted aminopyrazolo[3,4-b]pyridines. Acta Pol. Pharm. 2002, 59, 215-222.

7. Jiang, H.; Wang, X.-S. An Efficient Synthesis of Pyrazolo[3,4-b]pyridine Derivatives in Aqueous Media. J. Chin. Chem. Soc. 2007, 54, 1341-1345.

8. Bernardino, A.M.R.; Ferreira, V.F.; Fontoura, G.A.T.; Frugulhetti, I.C.P.P.; Lee, M.Y.; Romeiro, G.A.; Souza, M.C.B.; As, P.M. Synthesis of 4-Anilino-1H-Pyrazolo[3,4-b]pyridine Derivatives and their in vitro antiviral activity. J. Braz. Chem. Soc. 1996, 7, 273-277.

9. Slatt, J.; Romero, I.; Bergman, J. Cyanoacetylation of Indoles, Pyrroles and Aromatic Amines with the Combination Cyanoacetic Acid and Acetic Anhydride. Synthesis 2004, 16, 2760-2765.

10. Abdel-Motaleb, R.M.; Makhloof, A.A.; Ibrahim, H.M.; Elnagdi, M.H. Studies with azoles and benzoazoles: A novel simple approach for synthesis of 3-functionally substituted 3-acylindoles. J. Heterocycl. Chem. 2007, 44, 109-114.

11. Behbehani, H.; Ibrahim, H.M.; Makhseed, S. Studies with 3-oxoalkanonitriles: Reactivity of 3-oxo-(1-methylindoloyl)propanenitrile. Heterocycles 2009, 78, 3081-3090.

12. Khalil, K.D.; Al-Matar, H.M.; Al-Dorri, D.M.; Elnagdi, M.H. Studies with enaminones and enaminonitriles: Synthesis of 3-aroyl and 3-heteroaroyl-pyrazolo-[1,5-a]pyrimidines. Tetrahedron 2009, 65, 9421-9427.

13. Abdallah, T.A. Studies with Enamines and Azaenamines: Synthesis and Reactivity of 3-Dimethylamino-2-[(3-indolyl) carbonyl]propenonitrile. J. Heterocycl. Chem. 2007, 44, 961-965.

14. Sun, C.; Ji, S.-J.; Liu, Y. Facile synthesis of 3-(2-furanyl)indoles via a multicomponent reaction. Tetrahedron Lett. 2007, 48, 8987-8989.

15. Zhu, S.-L.; Ji, S.-J.; Su, X.-M.; Sun, C.; Liu, Y. Facile and efficient synthesis of a new class of bis(3'-indolyl)pyridine derivatives via one-pot multicomponent reactions. Tetrahedron Lett. 2008, 49, 1777-1781. 
16. Ibrahim, H.; Makhseed, S.; Abdel-Motaleb, R.M.; Makhloof, A.A.; Elnagdi, M.H. Utility of cyanoacetamides as precursors to pyrazolo[3,4- $d$ ]pyrimidine-4-ones, 2-aryl-6-substituted 1,2,3-triazolo[4,5- $d$ ]pyrimidines and pyrazolo[1,5-a]pyrimidine-3-carboxamides. Heterocycles 2007, 71, 1951-1966.

17. Quiroga, J.; Trilleras, J.; Gálvez, J.; Insuasty, B.; Abonía, R.; Nogueras, M.; Cobo, J.; Marchal, A. $\mathrm{C}$ - and N-cyanoacetylation of 6-aminopyrimidines with cyanoacetic acid and acetic anhydride. Tetrahedron Lett. 2008, 49, 5672-5675.

18. Behbehani, H.; Ibrahim, H.M.; Makhseed, S. Synthesis of 7-substituted pyrazolo[1,5-a]pyrimidine-3-carboxamides as potential non benzodiazepine hypnotics. ARKIVOC 2010, ii, 267-282.

19. Ghozlan, S.A.S.; Abdelhamid, I.A.; Ibrahim, H.M.; Elnagdi, M.H. Studies with 2-arylhydrazononitriles: A new convenient synthesis of 2,4-disubstituted-1,2,3-triazole-5-amines. ARKIVOC 2006, $X V, 53$.

20. Almousawi, S.M.; Moustafa, M.S. 2-Arylhydrazononitriles as building blocks in heterocyclic synthesis: A novel route to 2-substituted-1,2,3-triazoles and 1,2,3-triazolo[4,5-b]pyridines. Beilstein J. Org. Chem. 2007, 3, No.12.

21. Abdel-Motaleb, R.M.; Makhloof, A.A.; Ibrahim, H.M.; Elnagdi, M.H. Studies With 3-Functionally Substituted 2-Arylhydrazononitriles: A New Route to 3-Substituted-2-Arylhydrazononitriles, 4-Amino-pyrazole-5-carbonitriles, Azadienes and Cinnolines. J. Heterocycl. Chem. 2006, 43, 931-934.

22. Crystallographic data for 5a (ref. CCDC 804721) can be obtained on request from the director, Cambridge Crystallographic Data Center, 12 Union Road, Cambridge CB2 1EW, UK.

23. Crystallographic data for $\mathbf{1 0}$ (ref. CCDC 816562) can be obtained on request from the director, Cambridge Crystallographic Data Center, 12 Union Road, Cambridge CB2 1EW, UK.

24. Crystallographic data for 20f (ref. CCDC 815380) can be obtained on request from the director, Cambridge Crystallographic Data Center, 12 Union Road, Cambridge CB2 1EW, UK.

25. Crystallographic data for $\mathbf{2 3}$ (ref. CCDC 805282) can be obtained on request from the director, Cambridge Crystallographic Data Center, 12 Union Road, Cambridge CB2 1EW, UK.

Sample Availability: Samples of the compounds 3, 5, 15, 16 and 20 a-f are available from the authors.

(C) 2011 by the authors; licensee MDPI, Basel, Switzerland. This article is an open access article distributed under the terms and conditions of the Creative Commons Attribution license (http://creativecommons.org/licenses/by/3.0/). 